\title{
Neonatal Sinus Tachycardia, AE
}

National Cancer Institute

\section{Source}

National Cancer Institute. Neonatal Sinus Tachycardia, AE. NCI Thesaurus. Code C154932.

An adverse event in a newborn characterized by an abnormally high heart rate for age. 